\title{
Tuning optical properties of opal photonic crystals by structural defects engineering
}

\section{F. Di Stasio}

\section{Cucini}

\section{Berti}

D. Comoretto

comorett@chimica.unige.it

\section{A. Abbotto \\ L. Bellotto \\ N. Manfredi \\ C. Marinzi}

\author{
Dipartimento di Chimica e Chimica Industriale, Università degli Studi di Genova, via Dodecaneso 31, \\ 16146 Genova (Italy) \\ Dipartimento di Chimica e Chimica Industriale, Università degli Studi di Genova, via Dodecaneso 31, \\ 16146 Genova (Italy) \\ Dipartimento di Chimica e Chimica Industriale, Università degli Studi di Genova, via Dodecaneso 31, \\ 16146 Genova (Italy) \\ Dipartimento di Chimica e Chimica Industriale, Università degli Studi di Genova, via Dodecaneso 31, \\ 16146 Genova (Italy) \\ Dipartimento di Scienza dei Materiali e INSTM, Università di Milano-Bicocca, via Cozzi 53, 20125 \\ Milano (Italy) \\ Dipartimento di Scienza dei Materiali e INSTM, Università di Milano-Bicocca, via Cozzi 53, 20125 \\ Milano (Italy) \\ Dipartimento di Scienza dei Materiali e INSTM, Università di Milano-Bicocca, via Cozzi 53, 20125 \\ Milano (Italy) \\ Dipartimento di Scienza dei Materiali e INSTM, Università di Milano-Bicocca, via Cozzi 53, 20125 \\ Milano (Italy)
}

We report on the preparation and optical characterization of three dimensional colloidal photonic crystal (PhC) containing an engineered planar defect embedding photoactive push-pull dyes. Free standing polystyrene films having thickness between 0.6 and $3 \mu \mathrm{m}$ doped with different dipolar chromophores were prepared. These films were sandwiched between two artificial opals creating a PhC structure with planar defect. The system was characterized by reflectance at normal incidence angle ( $R$ ), variable angle transmittance ( $\mathrm{T}$ ) and photoluminescence spectroscopy (PL) Evidence of defect states were observed in $T$ and $R$ spectra which allow the light to propagate for selected frequencies within the pseudogap (stop band). [DOI: 10.2971/je0s.2009.09033]

Keywords: photonic crystals, dipolar chromophores, artificial opals

\section{INTRODUCTION}

Among PhC, i.e. materials possessing a periodic modulation of the dielectric constant on a length scale comparable to the wavelength of visible light, artificial opals are a simple and cheap playground to investigate optical effects [1, 2]. As a matter of fact, while traditional growth methods to prepare three dimensional PhC, like lithographic techniques, are time and energy consuming, self-assembly of building blocks like microspheres in the case of opals [3]-[11] is an interesting alternative process based on the spontaneous growth of stable well-defined structures, starting from elements which interact through non covalent bonds through quasi equilibrium steps, which intrinsically might repel defects. This technique is also scalable to wafer substrate and silicon technology [12]-[14]. The main drawback still remaining with opals is that their structure does not allow the formation of a complete photonic band gap (PBG). Only the inverse opal structure with the proper dielectric contrast allow opening a complete PBG [9]. Moreover, some care have to be used during the opal growth in order to reduce the role of possible defects like stacking faults [15]-[18]. In spite of these limitations, the advantages in the production of opals and their versatility to be engineered even in patterned substrates $[19,20]$ allows to simply test new ideas and concepts.

As already exploited in semiconductors technology, modulations and engineering of their properties is a key point. The main features of $\mathrm{PhC}$ depend on their structure and dielectric contrast [21]. Being the opal structure well defined (face centred cubic) with a unit cell length depending on sphere diameter, the main possibility to tune their properties is the modulation of the refractive index of composing materials. This approach has been widely exploited by several research groups by infiltrating into opal interstices a variety of materials ranging from metals, nanoparticles, inorganic and organic semiconductors (for a review of this fields see [22] and references therein). We exploited and investigated this latter effect by infiltrating opals with gold nanoparticles [23]-[25] and molecular [26] or polymeric semiconductors [27]. It should be noted that the use of organic chromophores as active materials for infiltration is rather unusual [28]-[36] and, when present, mostly limited to commercial dyes without a proper engineering of novel systems. 
A different approach to tune optical and photonic properties of PhC is represented by engineering structural defects [37]. In fact as atomic impurities change the potential in a semiconductor, structural defects have similar effects in PhC. Indeed, defects act as photonic dopants and depending on the structure, can trap the light inside the material. Point defects acts as optical cavities where the light is highly localized within the structure [38]. Linear defects work as waveguides in which the light is effectively conveyed through the structure by the PBG [38]-[40]. Planar defects shows both light localization and wave guiding effects [38]. The presence of a structural defect inside the periodical lattice of the dielectric constant gives rise to localized states [37, 41, 42]. Inside such localized states the electric field associated to the electromagnetic wave is concentrated [25]. This effect combined with a high quality factor of the cavity can provide an optical feedback in order to achieve laser emission [43]-[48] from localized states when an emitting materials is embedded in the planar defect $[49,50]$.

Different methods have been used to prepare defects inside opals and inverse opals like electron-beam lithography \& photolithography, spin-coating, sacrificial transfer \& excess layers, laser micro-annealing, nanoimprinting, Langmuir-Blodgett, multi-photon polymerization $[13,38,40,43,45,46,48,51]-[62]$. The typical optical signature of defect state in these structures is a minimum (maximum) on the top of the stop band observed in reflectance (transmittance) spectra. Recently, a very simple methods was used in order to obtain a planar structural defect in an opal structure. A free standing polymeric film doped with dyes few micron thick was sandwiched between two opals $[45,63,64]$. Even though such structures do not show any evidence of optical fingerprint of defect states, amplified spontaneous emission and lasing inside [45] or at the low energy edge $[63,64]$ of the photonic stop band was observed.

In this work we adopted the sandwich method to properly engineer opals with novel active materials embedded in structural defects for a possible lasing application. As active materials we have selected dipolar D- $\pi$-A dyes where a donor (D) and an acceptor (A) group are joined by a $\pi$ conjugated segment. The D- $\pi$-A molecular structure induces a push-pull effect of the electron density, with an intramolecular charge transfer occurring from the D to the A site of the molecule, thus providing polarity and polarizability to the system. These and similar dipolar dyes have been widely investigated for their non-linear optical properties, including two-photon absorption [65] and second-harmonic generation $[65,66]$.

We show here the use, for the first time, of these chromophores as active material in PhC structural defects with PL dye emission spectrum matching the opal stop band Evidence of defect states inside the photonic stop band was observed. The application of these dyes in the solid state is unprecedented in the literature. We also describe how these chromophores undergo, under specified conditions, fast photochemical degradation in spite of the good optical quality of the photonic crystal system.

\section{EXPERIMENTAL}

$\mathrm{T}$ and PL spectra were measured with optical set-ups based on fiber optical coupled Avantes 2048 compact spectrometer working in the $250-1100 \mathrm{~nm}$ range. The sample was mounted on a rotating stage allowing for $\mathrm{T}$ and PL measurements at different incidence or detections angle. The excitation for PL spectra was provided by an Oxxius (model 405-50-COL-PP) cw laser diode, $405 \mathrm{~nm}$, power $50 \mathrm{~mW}$.

Commercial polystyrene monodispersed microspheres (diameter $a=260 \mathrm{~nm}$, refractive index, $n_{P S}=1.59$; standard deviation $<5 \%$ ) water suspension $10 \%$ in volume (Duke Scientific) were used for opal preparation. The desired sample thickness (about $3 \mu \mathrm{m}$ thick) was obtained by properly diluting these solutions with de-ionized water and by growing the opal films using the meniscus technique at controlled temperature $\left(T=45 \pm 1^{\circ} \mathrm{C}\right)$ [10]. Sphere diameter was selected in order the stop band matches the emission spectrum of the dyes.

Free-standing films of carboxy-terminated polystyrene $\left(M_{w}=\right.$ 100.000, Sigma-Aldrich) blended to dyes (10:1 weight ratio) were drop-cast on glass substrates from solutions. After solvent evaporation, the polymeric film (thickness $0.6-5 \mu \mathrm{m}$ ) was peeled off from the substrate and sandwiched between opals thus creating the structural defect.

1-(4-NCetyl-4-pyridinium)-2-(N-methylpyrrol-2-yl) ethene bromide $\left(\mathrm{C}_{16 \mathrm{PEP}}{ }^{+}\right.$, Figure 1(a) inset) and 1-[4- $N-(3,7-$ dimethyloctyl)-4-pyridinium]-2-(N-methylpyrrol-2-yl) ethene triflate $\left(\mathrm{DMOPEP}^{+}\right.$Figure $1(\mathrm{~b})$ inset) were used as defect dopants. These dyes present a dipolar D- $\pi$-A structure where $\mathrm{A}$ is a $\pi$-deficient ( $\mathrm{N}$-alkylpyridinium) and $\mathrm{D}$ a $\pi$-excessive (pyrrole) heteroaromatic ring $\mathrm{C} 6 \mathrm{PEP}^{+}$and $\mathrm{DMOPEP}^{+}$ were synthesized by alkylation of 1-(pyrid-4-yl)-2-( $N$ methylpyrrol-2-yl) ethane [65] with hexadecyl bromide or 3,7-dimethyloctyl trifluoromethanesulfonate in dry toluene, respectively.

$\mathrm{C} 16 \mathrm{PEP}^{+}$exists in DMSO solution $\left({ }^{1} \mathrm{H}-\mathrm{NMR}\right)$ as a mixture 85:15 of trans and cis isomers (Figure 1(a) inset). The two isomers could not be separated by chromatography and therefore were used as a mixture for the defect doping. In contrast a DMSO solution $\left({ }^{1} \mathrm{H}-\mathrm{NMR}\right)$ of $\mathrm{DMOPEP}^{+}$shows the exclusive presence of the trans isomer.

These dipolar dyes have a saline nature and are usually not soluble (or slightly soluble) in organic solvents. Both dyes have been therefore functionalized at the pyridine nitrogen with a long alkyl chain in order to provide solubility in common organic solvents and then their processability and dispersion in polymers matrix (e.g., defect film preparation). The side functionality is a linear chain in the case of $\mathrm{C} 16 \mathrm{PEP}^{+}$, whereas a branched chain has been introduced in $\mathrm{DMOPEP}^{+}$. The nature of the linear and branched chains is the same used in common organic semiconductors such as polythiophenes and poly( $p$-phenylenevinylene).

Toluene was the solvent for the preparation of the DMOPEP ${ }^{+}$ based defect while chloroform and/or toluene were used for the $\mathrm{C}_{16} \mathrm{PEP}^{+}$doped defect. The polystyrene films doped with 
$\mathrm{DMOPEP}^{+}$appeared highly uniform whereas those containing $\mathrm{C}_{16} \mathrm{PEP}^{+}$showed a reduced optical quality due to phase segregation, which induces scattering.

\section{RESULTS AND DISCUSSION}

Figure 1 shows the absorption and PL spectra of $\mathrm{C}^{16} \mathrm{PEP}^{+}$ $\left(\mathrm{DMOPEP}^{+}\right)$in diluted toluene solution $(<0.01 \%$ in weight $)$. The insets show the chemical structure of the two molecules. $\mathrm{C}_{16} \mathrm{PEP}^{+}$spectra show an absorbance peak at $455 \mathrm{~nm}$ and a PL peak at $520 \mathrm{~nm}$; no structure is detected in these spectra As expected, no significant change in the absorption spectra is observed by modification of the side chain, being the $\pi$ framework unvaried. Interestingly, the PL peak of $\mathrm{DMOPEP}^{+}$ is shifted to lower energies by $40 \mathrm{~nm}(560 \mathrm{~nm})$. A slight increase of spectral broadening is observed as well.

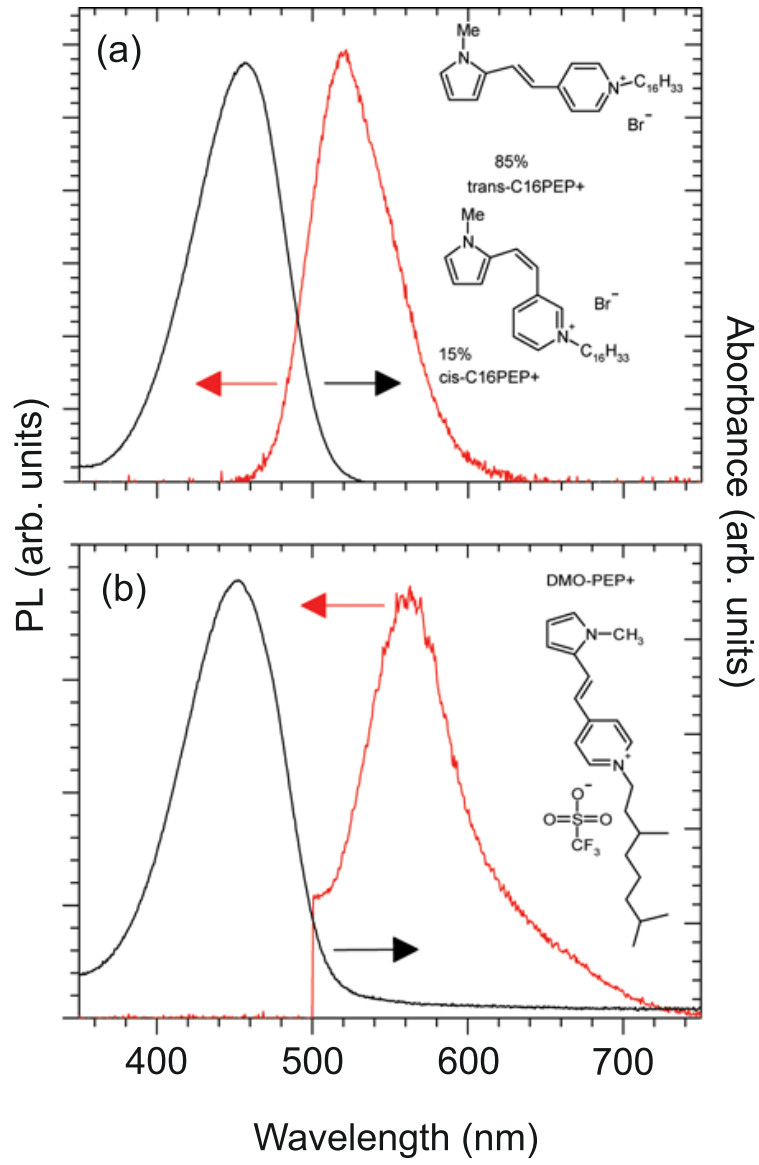

FIG. 1 (a) PL and absorbance spectra of C16PEP+ and (b) the DMOPEP+ in toluene $\left(25^{\circ} \mathrm{C}\right)$

The two opals composing the structures were characterized before the preparation of the sandwiches by normal incidence reflectance measurement (Figure 2(a) and (b) for $\mathrm{C}^{16} \mathrm{PEP}^{+}$ and $\mathrm{DMOPEP}^{+}$, respectively). Opals show the typical reflectance peak associated with the photonic pseudo gap (stop band) at $582 \mathrm{~nm}$. The presence of an extended interference fringes pattern in both spectral sides of the stop band provides information on the good optical quality of such opals. Interference fringes can also be used to calculate the thickness (d) of the samples once the effective refractive index $\left(n_{e f f}\right)$ of the opal is known by fitting transmittance spectra recorded at different incidence angles by the Bragg-Snell law (data not shown here for details see for instance [25]):

$$
d=\frac{N_{f}}{2 n_{e f f} \Delta \tilde{v}}
$$

where $N_{f}$ is the number of fringes in the $\Delta \tilde{v}\left(\mathrm{~cm}^{-1}\right)$ spectral range.

We found $d=2.2 \mu \mathrm{m}$ for the two opals with $\mathrm{C}_{16 \mathrm{PEP}^{+}}$and $d=2.8 \mu \mathrm{m}$ and $d=3.2 \mu \mathrm{m}$ for those with $\mathrm{DMOPEP}^{+}$.

The two defect layers were also characterized by $\mathrm{R}$ spectroscopy (see insets of Figure 2). A prominent interference fringe pattern was observed in the transparency window $(\lambda>$ $500 \mathrm{~nm}$ ). By assuming for these films a refraction index similar to that of a polystyrene matrix (1.6) we found a mean thickness value $d=1.5 \mu \mathrm{m}$ for $\mathrm{C} 16 \mathrm{PEP}^{+}$(Figure 2(a) inset) and $d=2.4 \mu \mathrm{m}$ for $\mathrm{DMOPEP}^{+}$(Figure 2(b) inset). The interference fringes and reflectance intensity are reduced in both spectra for wavelengths smaller than $500 \mathrm{~nm}$, due to the absorption of the emitting material in such spectral region.

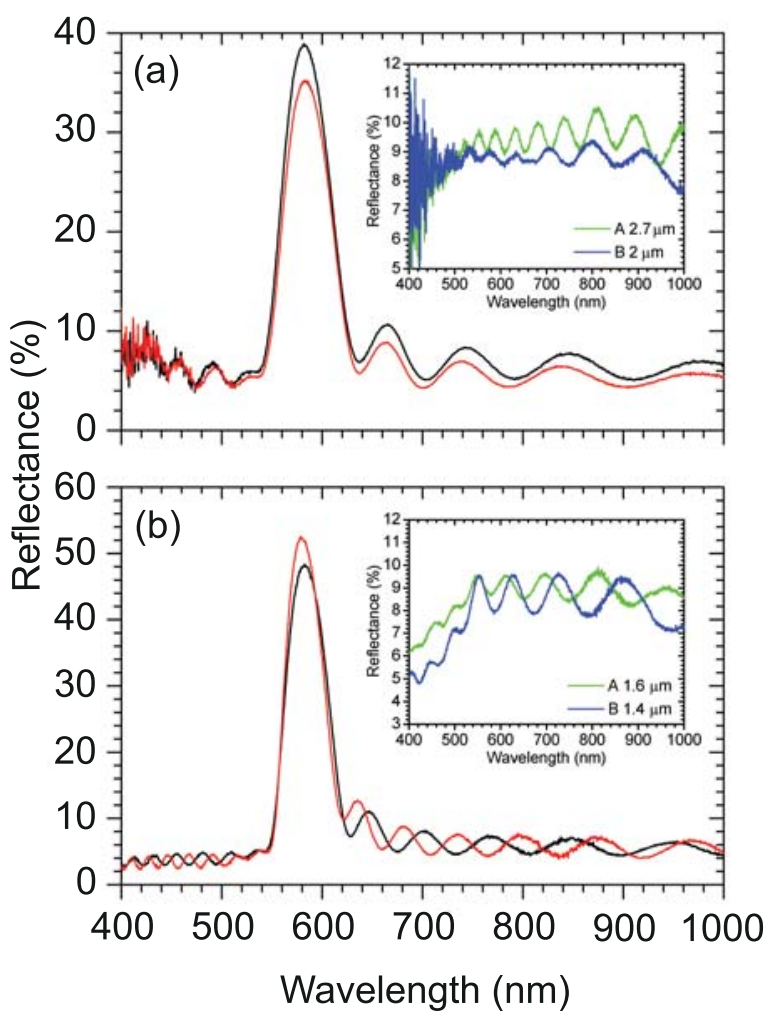

FIG. 2 (a) R spectra for opals composing the sandwich with planar defect containing C16PEP+ and (b) DMOPEP+. Insets ((a) and (b)) of R spectra of the planar defects.

After sandwiching opals with the defect layer, $\mathrm{R}$ spectra resulted strongly modified. Figures $3(\mathrm{a})$ and (b) show $R$ spectra of opals with structural defects containing $\mathrm{C}_{16} \mathrm{PEP}^{+}$and $\mathrm{DMOPEP}^{+}$as dopants, respectively. The presence of defects strongly affects the spectral shape of the stop bands: in both cases several minima appeared for wavelength values within the stop band. The number of these minima seems to be dependent on the probing spot position on the sandwich as well as on the defect thickness. We are currently investigating the effect of such parameters on the optical response of the system [67]. In any case, we notice that the presence of minima on top of opal stop band indicates an increased transparency of the photonic crystal. Such effect was expected 
as a consequence of the presence of a defect, i.e. the generation of allowed optical states inside a minimum of the density of photonic states (stop band). For such reason such minima can be recognized as a fingerprint of photonic defects. It is interesting to note that literature data for opals containing thin defects report the presence of a single minimum $[38,41,43,46,48,51]-[55,58,60]$. A possible rationale of such discrepancy could be related to the defect thickness that in those cases $[38,41,43,46,48,51]-[55,58,60]$ is much smaller than the cell length of the opal crystal, while in our work, is much larger thus allowing for several modes to propagate. It is worthwhile noting that for structural defects created with the sandwich technique, no defect modes in the optical spectra have been previously reported $[45,63,64]$, thus suggesting an high optical quality for our samples. According to our previous experience, lack of defects modes occurs when defect layer is strongly absorbing and scattering thus reducing the possibility of multiple reflections inside the sample or when opals are very thick.

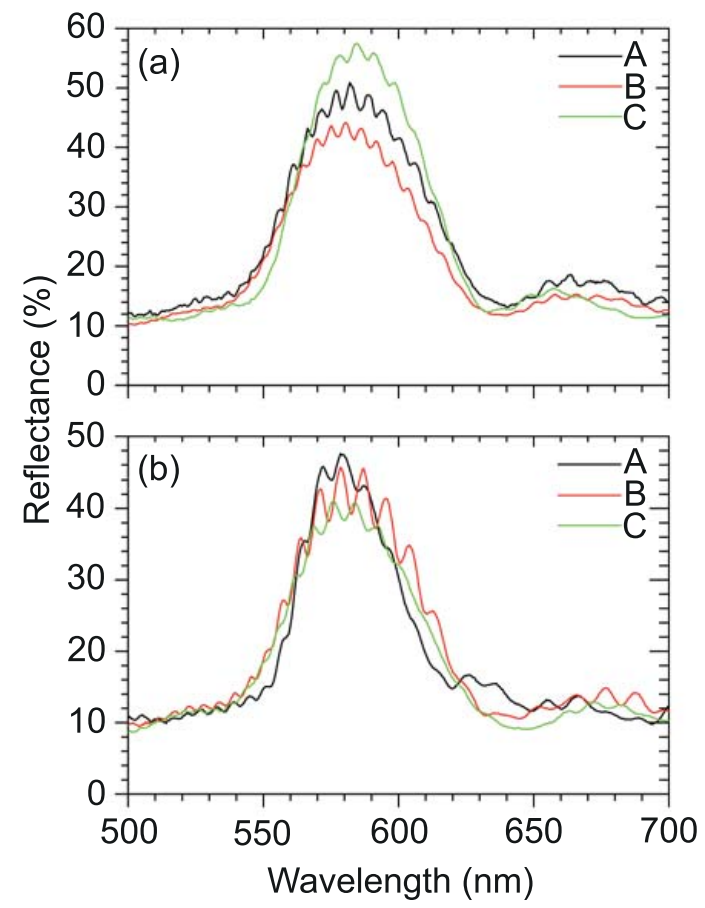

FIG. 3 (a) R spectra in different positions for the sandwich with C16PEP+ and (b) with DMOPEP+.

Once the optical response of the photonic structure was elucidated, we have investigated their emission properties. Unfortunately no PL signal was observed in opals despite solutions used to cast the films possess a bright emission. Indeed, we have observed that the PL signal is rapidly quenched during film drying suggesting a degradation or a change in the electronic structure of the dye. We have therefore decided to gain deeper insight in the nature of the dye transformation, in order to get clues for the design of new performing materials.

We first investigated the PL stability of the chromophores in different solvents. We prepared solutions of $\mathrm{C}^{26 \mathrm{PEP}^{+}}$ in chloroform acetonitrile and dimethylsulfoxide (DMSO). The change of absorption and PL spectra during three hours of continuous irradiation with a $405 \mathrm{~nm}$ laser excitation was monitored (Figure 4). After 3 hours of irradiation, the absorbance spectrum of $\mathrm{C} 6 \mathrm{PEP}^{+}$in chloroform is fully bleached. A significant effect is observed also for the PL spectrum, where two maxima (568 and $482 \mathrm{~nm}$ ) are observed in place of the one at $520 \mathrm{~nm}$ at $t=0$. This behaviour can be ascribed to the acid traces present in chloroform, which are known to decompose pyrrole derivatives. In fact, no degradation is observed in acetonitrile solutions. Unfortunately, in spite of such positive behaviour, we could not use this solvent for the defect preparation due to the low solubility of polystyrene. The degradation process is almost absent in DMSO solutions where only a partial bleaching was observed in the absorption spectra. Despite the photostability of $\mathrm{C}_{16} \mathrm{PEP}^{+}$in proper solvents, the emission is quenched in the solid state once the solvent is removed from these solutions.

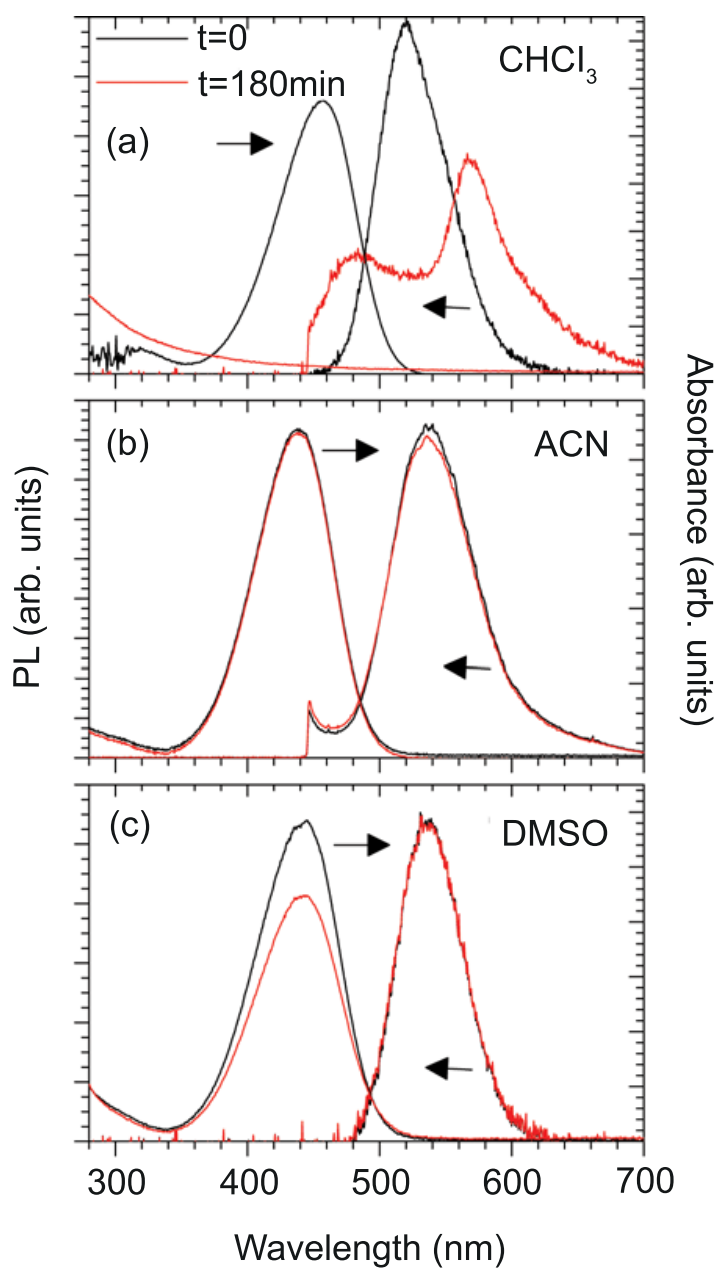

FIG. 4 (a) PL and absorbance spectra for diluted solutions of C16PEP+ in chloroform $\left(\mathrm{CHCl}_{3}\right)$, (b) acetonitrile ( $\mathrm{ACN}$ ), and (c) dimethylsulfoxide (DMSO).

Similar effects were also observed for $\mathrm{DMOPEP}^{+}$. The degradation process was investigated in the same solvent (toluene) used to prepare the defects (Figure 5). A significant change is observed in the absorbance spectra after $150 \mathrm{~min}$ of irradiation with the formation of a new shoulder at $524 \mathrm{~nm}$. No modification was observed in the PL spectra; however, PL bleaching during the solvent evaporation from the film was observed for this molecule as well.

The PL bleaching observed in the solid state for both dyes has likely a different nature from that occurring in solution and can be ascribed to intermolecular interactions which 
are known to provide similar effects [68]. We are currently working on the chemical design and synthesis of new hyperbranched substituted D- $\pi$-A dyes with enhanced stability and emitting properties in the solid state.

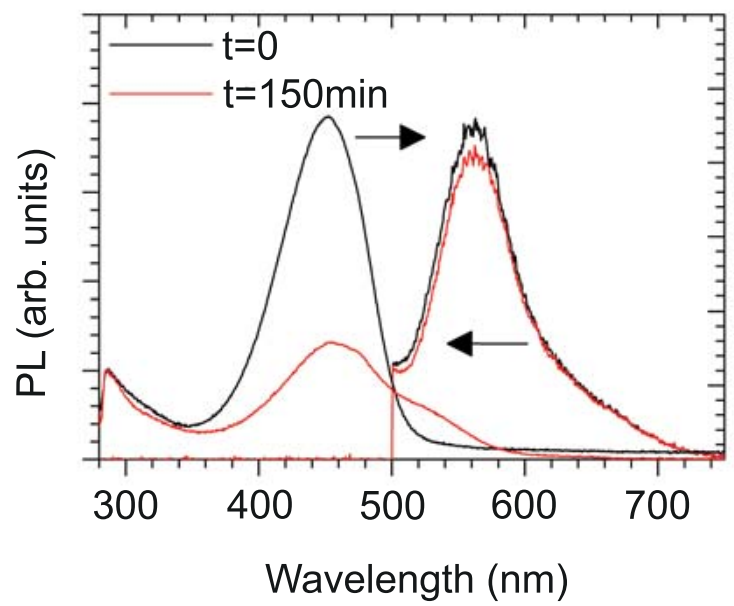

FIG. 5 PL and absorbance spectra for a diluted solution of DMOPEP+ in toluene.

\section{CONCLUSIONS}

We have prepared and characterized good optical quality opals. A polystyrene film doped with two saline dipolar chromophores was sandwiched between two opals thus creating a structural defect of the PhC. These new photonic structures show very different spectral features if compared to standard opals, being the stop band modified by the presence of several different minima in reflectance indicating an increased transparency. We tentatively assigned these structures to a spectroscopic signature of defect states within the photonic stop band. Unfortunately, it was impossible to characterize the photoluminescence spectrum of our defect states due to the PL bleaching in the solid state. A photochemical characterization of these dyes suggests that the observed PL quenching in the solid state is likely related to intermolecular interaction effects not occurring in solution.

\section{ACKNOWLEDGEMENTS}

We acknowledge helpful discussions with A. Painelli, F. Terenziani, and F. Marabelli. This research was supported by Ministero dell'Istruzione, dell'Università e della Ricerca (grants PRIN 2006031511 and FIRB RBNE033KMA).

\section{References}

[1] E. Yablonovitch, "Inhibited spontaneous emission in solid-state physics and electronics" Phys. Rev. Lett. 58, 2059 (1987).

[2] S. John, "Strong localization of photons in certain disordered dielectric superlattices" Phys. Rev. Lett. 58, 2486 (1987).

[3] D. J. Norris, E. G. Arlinghaus, L. Meng, R. Heiny, and L. E. Scriven, "Opaline photonic crystals: how does self-assembly work?" Adv. Mater. 16, 1393 (2004).

[4] Y. Xia, B. Gates, and Z. Y. Li, "Self-assembly approaches to threedimensional photonic crystals" Adv. Mater. 13, 409 (2001).
[5] J. E. G. J. Wijnhoven and W. L. Vos, "Preparation of photonic crystals made of air spheres in titania" Science 281, 802 (1998).

[6] A. A. Zakhidov, R. H. Baughman, Z. Iqbal, C. Cui, I. Khayrullin, S. 0. Dantas, J. Marti, and V. G. Ralchenko, "Carbon structures three-dimensional periodicity at optical wavelengths" Science 282, 897 (1998).

[7] D. J. Norris and Y. A. Vlasov, "Chemical approaches to threedimensional semiconductor photonic crystals" Adv. Mater. 13, 371 (2001).

[8] S. G. Romanov, T. Maka, C. M. S. Torres, M. Muller, and R. Zentel, "Light emission from thin opaline photonic crystals of low and high dielectric contrast" Synthetic Met. 124, 131 (2001).

[9] A. Blanco, E. Chomski, S. Grabtchak, M. Ibisate, S. John, S. W. Leonard, C. Lopez, F. Meseguer, H. Miguez, J. P. Mondia, G. A. Ozin, 0. Toader, and H. M. v. Driel, "Large-scale synthesis of a silicon photonic crystal with a complete three-dimensional bandgap near 1.5 micrometres" Nature 405, 437 (2000).

[10] P. Jiang, J. F. Bertone, K. S. Hwang, and V. L. Colvin, "Single-crystal colloidal multilayers of controlled thickness" Chem. Mater. 11, 2132 (1999).

[11] P. Jiang, J. F. Bertone, and V. L. Colvin, "A lost-wax approach to monodisperse colloids and their crystals" Science 291, 453 (2001).

[12] F. Jonsson, C. M. S. Torres, J. Seekamp, M. Schniedergers, A. Tiedemann, J. Ye, and R. Zentel, "Artificially inscribed defects in opal photonic crystals" Microelectron. Eng. 78-79, 429 (2005).

[13] T. A. Taton and D. J. Norris, "Device physics: Defective promise in photonics" Nature 416, 685 (2002).

[14] Y. A. Vlasov, X. Z. Bo, J. C. Sturm, and D. J. Norris, “On-chip natural assembly of silicon photonic bandgap crystals" Nature 414, 289 (2001).

[15] V. N. Astratov, A. M. Adawi, S. Fricker, M. S. Skolnick, D. M. Whittaker, and P. N. Pusey, "Interplay of order and disorder in the optical properties of opal photonic crystals" Phys. Rev. B 66, 165215 (2002).

[16] Y. A. Vlasov, V. N. Astratov, A. V. Baryshev, A. A. Kaplyanskii, 0. Z. Karimov, and M. F. Limonov, "Manifestation of intrinsic defects in optical properties of self-organized opal photonic crystals" Phys. Rev. E 61, 5784 (2000).

[17] Y. A. Vlasov, M. Deutsch, and D. J. Norris, "Single-domain spectroscopy of self-assembled photonic crystals" Appl. Phys. Lett. 76, 1627 (2000).

[18] J. F. Galisteo Lòpez and W. L. Vos, "Angle-resolved reflectivity of single-domain photonic crystals: Effects of disorder" Phys. Rev. E 66, 036616 (2002).

[19] C. A. Fustin, G. Glasser, H. W. Spiess, and U. Jonas, "Site-selective growth of colloidal crystals with photonic properties on chemically patterned surface" Adv. Mater. 15, 1025 (2003).

[20] X. Y. Ling, I. Y. Phang, W. Maijenburg, H. Schönherr, D. N. Reinhoudt, G. J. Vancso, and J. Huskens, "Free-standing 3D supramolecular hybrid particle structures" Angew. Chem. Int. Edit. 48, 983 (2009).

[21] J. D. Joannopulos, R. D. Meade, and J. N. Win, Photonic Crystals: Molding the Flow of the Light (Princeton University Press, Princeton, 1995).

[22] C. Lopez, "Materials aspects of photonic crystals" Adv. Mater. 15, 1679 (2003).

[23] D. Comoretto, V. Morandi, F. Marabelli, V. Amendola, and M. Meneghetti, "Optical effects in artificial opals infiltrated with 
gold nanoparticles" SPIE Proc. Ser. 6182, 61820D (2006).

[24] V. Morandi, F. Marabelli, V. Amendola, M. Meneghetti, and D. Comoretto, "Colloidal photonic crystals doped with gold nanoparticles; spectroscopy at optical switching properties" Adv. Funct. Mater. 17, 2770 (2007).

[25] V. Morandi, F. Marabelli, V. Amendola, M. Meneghetti, and D. Comoretto, "Light localization effect on the optical properties of opals doped with gold nanoparticles" J. Phys. Chem. C 112, 6293 (2008).

[26] M. Cucini, D. Comoretto, M. Galli, F. Marabelli, A. Abbotto, L. Bellotto, and C. Marinzi, "Emission properties of artificial opals infiltrated with a heteroaromatic quadrupolar dye" SPIE Proc. Ser. 6999, 69992D (2008).

[27] D. Comoretto, F. Marabelli, C. Soci, M. Galli, E. Pavarini, M. Patrini, and L. C. Andreani, "Morphology and optical properties of bare and polydiacetylenes-infiltrated opals" Synthetic Met. 139, 633 (2003).

[28] E. P. Petrov, V. N. Bogomolov, I. I. Kalosha, and S. V. Gaponenko, "Spontaneous Emission of Organic Molecules Embedded in a Photonic Crystal" Phys. Rev. Lett. 81, 77 (1998).

[29] M. Deutsch, Y. A. Vlasov, and D. J. Norris, "Conjugated-polymer photonic crystals" Adv. Mater. 12, 1176 (2000).

[30] N. Eradat, A. Y. Sivachenko, M. E. Raikh, Z. V. Vardeny, A. A. Zakhidov, and R. H. Baughman, "Evidence for braggoriton excitations in opal photonic crystals infiltrated with highly polarizable dyes" Appl. Phys. Lett. 80, 3491 (2002).

[31] N. Eradat, M. Wohlgenannt, Z. V. Vardeny, A. A. Zakhidov, and R. H. Baughman, "Studies of optical transitions related to piconjugated polymers and laser dyes infiltrated in opal photonic crystals" Synthetic Met. 116, 509 (2001).

[32] J.-C. Hong, J. H. Park, C. Chun, and D. Y. Kim, "Photoinduced tuning of optical stop bands in azopolymer based inverse opal photonic crystals" Adv. Funct. Mater. 17, 2462 (2007).

[33] M. N. Shkunov, Z. V. Vardeny, M. C. DeLong, R. C. Polson, A. A. Zakhidov, and R. H. Baughman, "Tunable, gap-state lasing in switchable directions for opal photonic crystals" Adv. Funct. Mater. 12, 21 (2002)

[34] R. C. Polson, A. Chipouline, and Z. V. Vardeny, "Random Lasing in $\pi$-Conjugated Films and Infiltrated Opals" Adv. Mater. 13, 760 (2001).

[35] R. C. Polson and Z. V. Vardeny, "Organic random laser in the weakscattering regime" Phys. Rev. B 71, 045205 (2005).

[36] P. Markowicz, C. Friend, Y. Shen, J. Swiatkiewicz, P. N. Prasad, 0. Toader, S. John, and R. W. Boyd, "Enhancement of two-photon emission in photonic crystals" Opt. Lett. 27, 351 (2002).

[37] J. D. Joannopoulos, P. R. Villeneuve, and S. Fan, "Photonic crystals: putting a new twist on light" Nature 386, 143 (1997).

[38] P. V. Braun, S. A. Rinne, and F. Garcia-Santamaria, "Introducing defects in 3D photonic crystals: state of the art" Adv. Mater. 18, 2665 (2006).

[39] Y. H. Ye, T. S. Mayer, I. C. Khoo, I. B. Divliansky, N. Abrams, and T. E. Mallouk, "Self-assembly of three-dimensional photoniccrystals with air-core line defects" J. Mater. Chem 12, 3637 (2002).

[40] Q. Yan, Z. Zhou, X. S. Zhao, and S.J. Chua, "Line defects embedded in three-dimensional photonic crystals" Adv. Mater. 17, 1917 (2005).

[41] J. F. Galisteo-Lopez, M. Galli, L. C. Andreani, A. Mihi, R. Pozas, M. Ocana, and H. Miguez, "Phase delay and group velocity determination at a planar defect state in three dimensional photonic crystals" Appl. Phys. Lett. 90, 101113 (2007).

[42] D. L. C. Chan, E. Lidorikis, and J. D. Joannopoulos, "Point defect geometries in inverted opal photonic crystals" Phys. Rev. E 71, (2005).

[43] E. Palacios-Lidon, J. F. Galisteo-Lopez, B. H. Juarez, and C. Lopez, "Engineered planar defects embedded in opals" Adv. Mater. 16, 341 (2004).

[44] Q. Yan, Z. Zhou, and X. S. Zhao, "Introduction of three-dimensional extrinsic defects into colloidal photonic crystals" Chem. Mater. 17, 3069 (2005).

[45] S. Furumi, H. Fudouzi, H. T. Miyazaki, and Y. Sakka, "Flexible polymer colloidal-crystal lasers with a light-emitting planar defect" Adv. Mater. 19, 2067 (2007).

[46] N. Tetreault, A. Mihi, H. Miguez, I. Rodriguez, G. A. Ozin, F. Meseguer, and V. Kitaev, "Dielectric planar defects in colloidal photonic crystal films" Adv. Mater. 16, 346 (2004).

[47] J. R. Lawrence, Y. Ying, P. Jiang, and S. H. Foulger, "Dynamic tuning of organic lasers with colloidal crystals" Adv. Mater. 18, 300 (2006).

[48] L. Wang, Q. Yan, and X. S. Zhao, "From planar defect in opal to planar perfect in inverse opal" Langmuir 22, 3481 (2006).

[49] S. Noda, A. Chutian, and M. Imada, "Trapping and emission of photons by a single defect in a photonic bandgap structure" Nature 407, 608 (2000).

[50] 0. Painter, R. K. Lee, A. Scherer, A. Yariv, J. D. O’Brien, P. D. Dapkus, and I. Kim, "Two-dimensional photonic band-gap defect mode laser" Science 284, 1819 (1999).

[51] R. Pozas, A. Mihi, M. Ocana, and H. Miguez, "Building nanocrystalline planar defects within self-assembled photonic crystals by spin-coating" Adv. Mater. 18, 1183 (2006).

[52] A. Arsenault, F. Fleischhaker, G. v. Freymann, V. Kitaev, H. Miguez, A. Mihi, N. Tetreault, E. Vekris, I. Manners, S. Aitchison, D. Perovic, and G. A. Ozin, "Perfecting imperfection-designer defects in colloidal photonic crystals" Adv. Mater. 18, 2779 (2006).

[53] Q. Yan, L. K. Teh, Q. Shao, C. C. Wong, and Y. M. Chiang, "Layer transfer approach to opaline hetero photonic crystals" Langmuir 24, 1796 (2008).

[54] N. Tetreault, A. C. Arsenault, A. Mihi, S. Wong, V. Kitaev, I. Manners, H. Miguez, and G. A. Ozin, "Building tunable planar defects into phoyonics crystals using polyelectrolyte multilayers" Adv. Mater. 17, 1912 (2005).

[55] F. Fleischhaker, A. C. Arsenault, J. Schmidtke, R. Zentel, and G. A. Ozin, "Spin-coating of designed functional planar defects in opal film: generalized synthesis" Chem. Mater. 18, 5640 (2006).

[56] N. Tetreault, H. Miguez, S. M. Yang, V. Kitaev, and G. A. Ozin, "Refractive index patterns in silicon inverted colloidal photonic crystals" Adv. Mater. 15, 1167 (2003).

[57] Y. Jun, C. A. Leatherdale, and D. J. Norris, "Tailoring air defects in self-assembled photonic bandgap crystals" Adv. Mater. 17, 1908 (2005).

[58] F. Fleischhaker, A. C. Arsenault, V. Kitaev, F. C. Peiris, G. v. Freymann, I. Manners, R. Zentel, and G. A. Ozin, "Photochemically and thermally tunable planar defects in colloidal photonic crystals" J. Am. Chem. Soc. 127, 9318 (2005).

[59] Q. Yan, A. Chen, S. J. Chua, and X. S. Zhao, "Incorporation of point defects into self-assembled three-dimensional colloidal crystals" Adv. Mater. 17, 2849 (2005).

[60] P. Massé, S. Reculusa, K. Clays, and S. Ravaine, "Tailoring planar defect in three-dimensional colloidal crystals" Chem. Phys. Lett. 
422, 251 (2006).

[61] Q. Yan, L. Wang, and X. S. Zhao, "Artificial defect engineering in three-dimensional colloidal photonic crystals" Adv. Funct. Mater. 17,3695 (2007).

[62] W. Lee, S. A. Pruzinsky, and P. V. Braun, "Multi-photon polymerization of waveguide structures within three-dimensional photonic crystals" Adv. Mater. 14, 271 (2002).

[63] J. Feng, S. Yang, D. Xian-Zi, C. Wei-Qiang, and D. Xuan-Ming, “Amplified spontaneous emission from dye-doped polymer film sandwiched by two opal photonic crystals" Appl. Phys. Lett. 91, 031109 (2007).

[64] F. Jin, C.-F. Li, X.-Z. Dong, W.-Q. Chen, and X.-M. Duan, "Laser emission fron dye-doped polymer film in opal photonic crystal cavity" Appl. Phys. Lett. 89, 241101 (2006).
[65] A. Abbotto, L. Beverina, S. Bradamante, A. Facchetti, C. Klein, G. A. Pagani, M. Redi-Abshiro, and R. Wortmann, "A distintive example of the cooperative interplay of structure and environment in tuning of intramolecular charge transfer in second-order nonlinear optical chromophores" Chem. Eur. J. 9, 1991 (2003).

[66] G. Archetti, A. Abbotto, and R. Wortmann, "Effect of polarity and structural design on molecular photorefractive properties of heteroaromatic-based push-pull dyes" Chem. Eur. J. 12, 7151 (2006).

[67] F. Di Stasio, M. Cucini, L. Berti, D. Comoretto, M. Galli, F. Marabelli, A. Abbotto, L. Bellotto, C. Marinzi, S. Gardin, T. Dainese, R. Signorini, and R. Bozio, in preparation

[68] M. Pope and C. E. Swenberg, Electronic processes in organic crystals and polymers (Oxford University Press, New York, 1999). 